\title{
Los profesionales de la psicología de la educación en el siglo XXI: Un escenario complejo
}

\section{Educational psychology professionals in the 21st Century: A complex scenario}

Fecha de recepción: 29-03-2017

Fecha de aceptación: 16-05-2017
Francisco Pérez González

Profesor Titular del Departamento de Psicología Evolutiva y de la Educación.

Universitat de València

Rafael García-Ros

Profesor Titular del Departamento de Psicología Evolutiva y de la Educación.

Universitat de València

\section{resumen/abstract:}

Se analizan las problemáticas actuales de los psicólogos de la educación en relación a sus competencias profesionales, formación especializada y procesos de acreditación para el ejercicio profesional. Se destaca que las figuras de Psicólogo Especialista en Psicología Clínica y de Psicólogo General Sanitario han generado profundos desequilibrios en las demandas de formación de grado y de postgrado hacia el ámbito clínico, dejando en un segundo plano el ámbito formativo y profesional del Psicólogo de la Educación. Se destaca la necesidad de disponer de un máster específico de especialización, consensuado entre todas las universidades públicas, que permita garantizar el desarrollo de las competencias profesionales del psicólogo educativo en contextos educativos formales. Se propone la creación de servicios de orientación multidisciplinares en que los psicólogos educativos constituyan un componente esencial de los mismos. Estas propuestas podrían implicar replantear la estructura actual del Master en Psicología General Sanitaria, facilitando la cohesión y reduciendo las tensiones actuales entre los ámbitos disciplinares y profesionales de la psicología.

The article analyzes the current problems of educational psychologists in terms of their professional competences, specialized training, and accreditation processes for professional practice. It highlights that the figures of the Specialist Psychologist in Clinical Psychology and General Healthcare Psychology have produced pronounced imbalances in the demands for degree and post-graduate training toward the clinical realm, relegating the training and professional area of Educational Psychologists to a secondary role. The article indicates the need for a specific masters specialization, through consensus among all the public universities, which would make it possible to guarantee the development of the professional competences of the educational psychologist in formal educational contexts. The creation of multidisciplinary guidance services is proposed, where educational psychologists would be key components. These proposals could involve redesigning the current structure of the Master in General Healthcare Psychology, facilitating cohesion and reducing the current tensions between the disciplinary and professional areas of psychology.

\section{palabras clave/keywords:}

Psicología de la educación, formación especializada, rol del psicólogo escolar, intervención psicoeducativa, acreditación profesional

Educational psychology, specialized training, role of the school psychologist, psychoeducational intervention, professional accreditation 


\section{I.- Introducción: Situar el problema}

Nunca antes en nuestro país se había suscitado una discusión tan apasionada sobre la orientación educativa, sus profesionales, los requisitos de formación y competencias requeridas en su desempeño, así como sobre los mecanismos dirigidos a la acreditación y acceso al ejercicio de la profesión. Simultáneamente, tal como se destaca en el capítulo introductorio de este monográfico, la regulación profesional del Psicólogo General Sanitario ha acentuado la asimetría entre las áreas profesionales de la psicología, generando numerosos debates y controversias sobre sus repercusiones en la identidad profesional y en la percepción social de la psicología en su conjunto, e incluso sobre el desamparo y abandono en que pueden quedar otras especializaciones consolidadas como la Psicología de la Educación. Estas cuestiones exigen abrir un proceso de reflexión y debate en nuestra área, e intentar dar una respuesta consensuada a las mismas desde el mundo académico y profesional.

Aunque la presencia de los psicólogos de la educación en contextos escolares goza de una amplísima tradición, que en nuestro país se inició con mucha anterioridad a la LOGSE (Ley General de Educación, 1970; Servicios Provinciales de Orientación Escolar y Profesional, 1977; Equipos Multiprofesionales, 1982), es a partir de su promulgación en 1990 cuando se inicia la consolidación definitiva del ámbito profesional en el que desarrollan sus funciones estos profesionales, es decir, el sistema educativo no universitario. Por otro lado, a lo largo de estos años se han ido modificando los requisitos formativos de acceso a la misma (en la actualidad haber cursado el Master de Formación de Profesorado de Educación Secundaria), los procedimientos de acceso en la función pública (concursos de méritos y sistema de oposiciones) y las denominaciones de estos profesionales (Profesor Especialista en Psicología y/o Pedagogía, Psicopedagogos y, en la actualidad, Orientadores).

Por otro lado, pese a que la Psicología la Educación constituye uno de los ámbitos profesionales de la psicología con mayor tradición y reconocimiento social, en nuestro país sólo existen actualmente dos profesiones reguladas que estipulen como requisito previo disponer de la licenciatura o del grado de Psicología: el Psicólogo Especialista en Psicología Clínica (al que se accede a través del PIR) y el Psicólogo General Sanitario (cuyo requisito es haber desarrollado el master de Psicólogo General Sanitario -MPGS). Y, dado el carácter también profesionalizante de la licenciatura y del grado en Psicología, quienes disponen de estas titulaciones pueden trabajar en el resto de ámbitos profesionales, hayan cursado o no formación de postgrado.

En este sentido, el reconocimiento de la psicología como profesión sanitaria y la reciente creación del MPGS como vía de acceso a la misma, ha generado una importantísima reorientación de las demandas de formación hacia el ámbito clínico y ha dejado en un segundo plano otros ámbitos profesionales y especializaciones. Por otro lado, aunque el Espacio Europeo de Educación Superior establecía la necesidad de contar con un máster específico de especialización que permitiera garantizar la cualificación profesional del Psicólogo Educativo, en nuestro país la única opción actual es cursar el máster de Formación del Profesorado de Secundaria para trabajar como tal en los contextos formales, sin contar con un máster específico en Psicología de la Educación. Y, si en algo hay un acuerdo gene- 


\section{dossiert}

ralizado entre todos los agentes implicados -Universidad, Colegio Profesional, Psicólogos Educativos-, es en considerar que el actual Máster de Formación del Profesorado no capacita a los potenciales profesionales de la orientación educativa en la gran diversidad de tareas de prevención, evaluación e intervención que se les demandan en los contextos escolares. Además, tal como se destaca en la introducción de este monográfico, esta última cuestión se agrava todavía más cuando el marco legal vigente permite que los graduados en cualquier otra titulación puedan optar a los puestos de trabajo de los servicios de orientación en los centros educativos. Basta para ello haber cursado la especialidad de orientación educativa del citado Máster, lo que cuestiona, todavía en mayor grado, que se pueda ofrecer desde el modelo de formación actual una respuesta ajustada al nivel de competencias requerido para desempeñar este puesto de trabajo y responder eficazmente a las demandas de familias, estudiantes, profesorado y equipos directivos de los centros educativos.

Estas consideraciones iniciales nos hacen plantearnos algunas cuestiones y reflexiones que queremos compartir en estas páginas. A modo de ejemplo, (1) ¿habría que clarificar y definir con mayor precisión el nivel de competencias, así como la amplitud y alcance de las actividades profesionales propias del psicólogo de la educación?; (2) ¿la forma de acceso actual al puesto de trabajo de psicólogo de la educación garantiza la adquisición de estas competencias o necesita ser profundamente reconsiderada?, (3) ¿se debieran redefinir y/o diversificar más los itinerarios formativos en psicología, especialmente en los títulos de postgrado sean o no profesionalizantes (aunque también en los títulos de grado)? Dicho de otra manera, ¿la Psicología de la Educación debe ser una profesión regulada más al igual que el Psicólogo Clínico o el Psicólogo General Sanitario (y, por extensión, quizás a otros ámbitos de la psicología)? Para tratar de contestar a estas cuestiones consideramos necesario abordar, en primer lugar, cuáles deben ser las competencias académicas y profesionales de los psicólogos de la educación, cuáles debieran ser las funciones/demandas a las que responder y cuáles deberían ser las formas de acceso a la misma.

Estamos convencidos de que mejorando la formación y las competencias de los psicólogos de la educación, definiendo adecuadamente sus funciones y tareas, colaborando con las múltiples iniciativas surgidas del mundo profesional y compartiendo nuestras reflexiones podremos contribuir a la consolidación de la profesión y colaborar, junto al resto de profesionales presentes en los contextos escolares, a la mejora de la calidad de la educación.

\section{II.- Desarrollo: ¿A qué nos referimos?}

Los análisis más recientes sobre la situación actual de los profesionales de la psicología de la educación en nuestro país (p.e., Fernández, 2011; Peretó y de Vicente, 2014; Pérez-Solís, 2011) destacan las enormes diferencias organizativas entre los servicios de orientación de las diferentes Comunidades Autónomas (equipos de sector, departamentos de orientación), así como en las características del modelo de intervención que asumen (p.e., priorización de funciones), en la composición de los servicios (número y formación inicial de los profesionales implicados), así como en las regulaciones específicas de las Administraciones Educativas en los diferentes territorios. Estas cuestiones han provocado que las prácticas profesionales de los psicólogos de la educación sean, en muchos casos, heterogéneas. 
Adicionalmente, la prestación de servicios en las diferentes etapas -y modalidades- educativas no universitarias (Infantil/Primaria, Secundaria, Centros Específicos de Educación Especial) también ha generado notables diferencias en el desarrollo y priorización de las funciones de estos profesionales. Y a esto, añadir las diferencias propias en el desempeño específico del puesto de trabajo por cada profesional en función de su perspectiva y enfoque conceptual, de su formación inicial y de sus experiencias de formación continua. A modo de ejemplo, sólo en la Comunidad Valenciana trabajan alrededor de 1400 orientadores distribuidos entre los Servicios Psicopedagógicos Escolares de sector (SPEs) y centros de Educación Especial (399), los Departamentos de Orientación en los Institutos de Enseñanza Secundaria (515) y la red de Gabinetes Psicopedagógicos Municipales (alrededor de 470). Por otro lado, también conviene destacar que la mayoría de estos profesionales desarrollan procesos de orientación más generales (p.e., asesoramiento vocacional, asesoramiento al profesorado en la acción tutorial), mientras que una minoría llevan a cabo intervenciones más especializadas -Equipos técnicos especializados- relacionadas con el diagnóstico e intervención en trastornos del desarrollo y dificultades del aprendizaje, en el ámbito de las discapacidades físicas y/o sensoriales, así como en el ámbito emocional y comportamental.

Por supuesto, no queremos obviar que numerosos psicólogos de la educación desarrollan sus funciones en otras áreas y contextos diferentes al escolar (p.e., organizacional y comunitario), demostración evidente de la utilidad y relevancia de los conocimientos de la Psicología de la Educación. Atendiendo a estas consideraciones, necesariamente debemos ampliar los escenarios y ámbitos en los que puede ejercer su trabajo este profesional. En este sentido, la consulta del Portal de Salidas Profesionales de la Universidad de Valencia (http://www.uv.es/uvweb/estudiants-uv/es/insercion-profesional/orientacion-asesoramiento/salidas-profesionales-1285927894719.htm), permite constatar el extenso repertorio de profesiones en las que encontramos a los psicólogos educativos, junto a otros profesionales con trayectorias formativas muy diferentes. Entre otras, además de las de Psicólogo/a educativo, destacar profesiones relacionadas con el asesoramiento y orientación psicosocial (psicólogo/a de la intervención comunitaria y orientador profesional), con la docencia (profesor/a de Enseñanza Secundaria, Profesor/a de Formación de personas adultas), relacionadas con la intervención con familias y menores en situación de riesgo (técnicos especialistas en atención al menor, psicólogo especialista en el apoyo a familias e infancia, mediador familiar) o con el ámbito de la gestión y tecnología educativa (director/a de centros educativos y de escuelas taller, gestor de formación, especialista en diseño instruccional y metodología de enseñanza). Todas estas profesiones tienen como denominador común la aplicación de los conocimientos psicológicos y educativos en el desempeño profesional, siendo conscientes de que la adquisición del conjunto de competencias implicadas en el desarrollo efectivo de los mismos puede derivarse de trayectorias formativas y profesionales claramente diferenciadas.

\section{1.- Compartir el espacio profesional, ¿un problema?}

En primer lugar, consideramos que resulta oportuno abordar la cuestión de la denominación de los profesionales de la psicología de la educación que desarrollan su actividad en contextos educativos formales. Desde nuestra perspectiva, queremos hacer constar esta cuestión

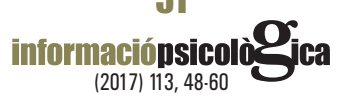




\section{dossier}

porque nos referimos al psicólogo escolar como un psicólogo de la educación que desarrolla sus funciones en los contextos escolares, familiares y socio-comunitarios con el objeto de intervenir - directa o indirectamente- en la mejora del desarrollo y del aprendizaje de los sujetos (Fagan y Wise, 1994, 2000; ISPA, 2002; Skalski, Minke, Rossen, Cowan, Kelly, Armistead \& Smith, 2015). En definitiva, lo que caracteriza a estos profesionales de la psicología frente a otras especialidades es, en primer lugar, el contexto de intervención y, en segundo lugar, la aplicación de los conocimientos psicológicos y educativos para la mejora de la calidad de los centros donde interviene (Gilman, Misall, \& Macks, 2011). La configuración de este espacio profesional es lo que denominamos psicología escolar y/o intervención psicoeducativa (Coll, 1988, 1989, 1996).

La psicología escolar es en la actualidad una especialidad reconocida en el campo psicológico aplicado que cuenta con directrices en cuanto a la formación inicial y de especialización de los profesionales así como las diversas vías de acreditación de la formación en diferentes países (p.e., Estados Unidos, Francia). Sin embargo, en España está regulada la profesión con la denominación de "Orientación Educativa", con un conjunto de actividades profesionales vinculadas en exclusiva a la misma, así como con unos requisitos formativos y de acceso específicos -titulación de Grado y Máster de Enseñanza Secundaria (especialidad de Orientación Educativa)-.

En cualquier caso, no queremos generar una polémica sobre la denominación de estos profesionales -psicólogos educativos, psicólogos escolares, orientadores-, que requeriría un análisis interesante pero con unos objetivos diferentes. Por tanto, vamos a utilizar indistintamente la denominación de psicólogo educativo, profesional de la psicología de la educación o psicólogo escolar en la medida que resultan los más utilizados en nuestro contexto y nos permite identificar, sin equívocos, al profesional al que hacemos referencia. Por otro lado, asumimos el término "orientador/a" en la medida que se corresponde con la denominación oficial actual en nuestro país del puesto de trabajo desempeñado por los psicólogos educativos que desarrollan su actividad profesional en los contextos educativos formales. Somos conscientes, no obstante, de que la denominación orientador (así como otras terminologías precedentes) ha podido contribuir en gran manera a la pérdida de la identidad profesional de los psicólogos educativos, de que en la actualidad la gran mayoría de los orientadores no disponen de titulación en psicología, y de que las funciones que desarrollan no siempre se corresponden (o al menos no cubren todo el espectro y alcance) con las de un psicólogo de la educación. Esta es una de las razones por las que también resulta pertinente reflexionar sobre el rol, los procesos formativos (formación requerida de grado y postgrado) y requisitos de acceso a la profesión, cuestiones a todas luces de actualidad.

En este sentido, y atendiendo a la composición de los Equipos de Sector y Departamentos de Orientación, se constata que junto a los psicólogos existe una amplia mayoría de profesionales con perfiles académicos diferentes que comparten este espacio profesional. Este hecho tiene, sin duda alguna, notables repercusiones a la hora de desarrollar las funciones propias del orientador en la medida que, en función de su correspondiente formación del grado, disponen de una preparación más amplia y ajustada para algunas funciones, una clara tendencia a priorizarlas de forma diferencial en su trabajo e, incluso, diferencias muy nota- 


\section{dossier}

bles en las estrategias y herramientas de intervención que utilizan, así como en la forma de dar respuesta a las demandas de los diferentes agentes educativos. No ponemos en duda que todos los candidatos pueden tener el potencial para convertirse en "buenos orientadores", pero cada uno va a requerir diferentes niveles de formación para desarrollar las habilidades y competencias requeridas (y socialmente repetidamente demandadas) por este puesto de trabajo.

Este hecho tiene consecuencias muy importantes desde el momento en que el perfil profesional y la delimitación de funciones para la orientación educativa es único, atribuyéndole las mismas funciones con independencia de la formación inicial de los profesionales que acceden a este puesto de trabajo. Esta es, sin duda, una de las razones por las que se constatan grandes diferencias entre orientadores en el desempeño de las funciones y tareas propias del puesto, que responden no sólo a las diferencias del entorno de trabajo sino también a las diferencias en las competencias básicas adquiridas en el proceso de formación universitaria, que no son compensadas en modo alguno en la propuesta formativa actual del master de profesorado de Secundaria. Una de las consecuencias de esta situación es que profesionales con perfiles formativos diferentes, competencias también diferentes, comparten el mismo espacio profesional y deben desarrollar, por tanto, las mismas funciones.

Por estas razones, entre las propuestas de mejora que consideramos urgentes para consolidar la figura del psicólogo de la educación, siempre partiendo de las necesidades actuales del sistema educativo (estudiantes, profesores y familias), está la de definir con claridad sus competencias, el nivel requerido de desarrollo en las mismas, así como el alcance de sus funciones y tareas específicas en el desempeño de la profesión. No hay que olvidar que los aspectos relacionados con la intervención del Psicólogo de la Educación - ¿qué hace?, ¿dónde interviene?, ¿cuáles deben ser sus funciones y prioridades?, ¿cómo hacerlo?, etc.- se desarrollan en estrecha interacción y se modifican en la medida en que el sistema educativo (la sociedad en su conjunto) cambia. Estos cambios son especialmente notables en momentos de Reformas Educativas como el actual que, sin duda alguna, va a modificar el marco y los condicionantes de la intervención de estos profesionales en un futuro próximo. Dicho de otro modo, el trabajo de los psicólogos educativos está mediatizado por los modelos de intervención definidos en el marco legislativo, las percepciones que de sus funciones tienen los agentes implicados - profesores, familias y estudiantes-, su nivel de competencias académicas y profesionales, las concepciones sobre la enseñanza y sobre cómo intervenir para intentar solucionar los problemas que se plantean en los procesos de enseñanza-aprendizaje.

En el marco de este análisis y discusión de la situación, resulta especialmente relevante recordar las conclusiones del I Encuentro Nacional de Profesionales de la Psicología de la Educación celebrado en Barcelona (2009), el Acuerdo sobre la Inserción del Psicólogo Educativo en el Sistema de Educación Español no Universitario celebrado en Madrid (2010) y el Manifiesto en defensa de la profesionalidad de la Orientación Educativa (2011) suscrito por las Facultades de Psicología del Estado y el Consejo General de Colegios Oficiales de Psicólogos. En este último se adquirió el compromiso de crear un máster específico de Psicología de la Educación centrado en la capacitación profesional para ejercer en contextos educativos, dado que el actual máster de Formación del Profesorado no es, en modo alguno, 
el medio adecuado para formar a estos profesionales. Compartimos estas ideas y deseos de todos los implicados en la mejora de la calidad de la educación, y de forma más directa en la figura del psicólogo educativo, y las hacemos nuestras al reivindicar su importancia y replantear su formación, sus competencias y los procedimientos de acceso al ejercicio de la profesión.

\section{2.- La consolidación del espacio profesional: ¿dónde está el problema?}

Uno de los problemas que está incidiendo en la consolidación del espacio profesional de los psicólogos de la educación hay que situarlo en la heterogeneidad de bagajes competenciales psicológicos y educativos de base de los profesionales que trabajan en los diferentes servicios de orientación, en la diversidad de funciones que desempeñan y en la priorización de las mismas, así como en las grandes diferencias en los modelos de prestación y en la organización de servicios. Estas cuestiones, entre otras, dificultan disponer de una visión universalmente aceptada de la profesión.

Por otro lado, también sabemos que aunque las administraciones educativas regulan unas funciones que (al menos teóricamente) conllevan el desarrollo de unas determinadas tareas, esto no significa que todos los profesionales las lleven a cabo en su conjunto ni que las prioricen del mismo modo y, mucho menos, que las realicen con estándares de calidad homogéneos. Todas estas cuestiones todavía resultan más complejas si las propias administraciones son las que priorizan, con distintos criterios y por distintos medios, las tareas a desarrollar, limitando otras actuaciones o ubicándolas en un plano secundario. Adicionalmente, otro problema fundamental es la ingente cantidad de funciones asignadas a estos profesionales, que provoca igualmente la necesidad de que el profesional intervenga en un abanico muy amplio de aspectos (p.e., dificultades del aprendizaje, altas capacidades, trastornos del desarrollo, problemas conductuales, de rendimiento y abandono académico temprano, prevención del suicidio, trastornos y problemas emocionales, etc.), cuestión que nos introduce en la problemática no sólo de la formación inicial de grado y postgrado, sino también de la articulación y definición de políticas de formación continua más eficaces, dado que esta cuestión no puede depender de la responsabilidad estrictamente individual de los profesionales.

Otro reto importante para la consolidación del espacio profesional de la psicología de la educación radica en la necesidad de asumir un marco de intervención comunitario más amplio. Es decir, el psicólogo educativo debe desarrollar sus funciones en un contexto comunitario que abarca los centros escolares, el contexto social próximo, así como la coordinación e interacción continua con los distintos agentes con implicación directa o indirecta en la educación. Entre las razones que justifican esta cuestión destaca el hecho de que una gran parte de las demandas y de los problemas que deben afrontar los profesionales no tienen solución desde los centros escolares sin la implicación de otras instancias (p.e., absentismo, relaciones familiares, trastornos del comportamiento, grupos de especial vulnerabilidad social, etc.). Todo ello también implica la exigencia de formación específica y de coordinación estrecha con otros servicios y entidades públicas y privadas.

Por ello, para llevar a cabo la consolidación del espacio profesional se requiere un acuerdo general acerca de la práctica, del rol y funciones que deben desarrollar estos profesionales, 
de los modelos de prestación de servicios más eficaces así como las exigencias en cuanto a su formación académica (psicológica y educativa) y práctica. Los programas de formación académica de los psicólogos educativos deben cambiar y adaptarse mejor a las necesidades planteadas por estos retos profesionales y por las demandas efectuadas por los distintos agentes implicados. Para ello, las propuestas de formación deberán enfatizar la formación en competencias que no son suficientemente desarrolladas en la formación de grado y que exigen la formación en postgrados que garanticen su adquisición para el desarrollo de las funciones que le son propias.

Sin entrar en este momento en la discusión acerca de cuáles deberían ser estas competencias, compartimos la idea expresada por Cesar Coll en relación a la relevancia que debe tener la psicología de la educación cuando afirma que "la psicología escolar no puede limitarse a las aportaciones de la psicología de la educación, aunque son precisamente estas aportaciones las únicas que pueden constituir el núcleo vertebrador de la intervención psicológica en la escuela" (Coll, 1988:320). En este sentido, cabe destacar la relevancia de las aportaciones de la psicología de la educación y de la instrucción como garantía de disponer de una sólida formación para poder ejercer el asesoramiento e intervención adecuado en cada caso, contando con la amplia diversidad de necesidades educativas especiales a las que se debe dar respuesta. Los programas de formación de los profesionales deberán enfatizar la importancia de esta disciplina educativa dada la necesidad de comprometer más si cabe, la psicología escolar, la práctica educativa, con la psicología de la educación. Y todo ello junto a una sólida formación en los trastornos del desarrollo, las dificultades del aprendizaje, la evaluación psicológica y educativa que permita identificar y determinar con precisión las necesidades de los estudiantes, y dar una respuesta educativa adecuada a las mismas. Este contexto favorecedor del cambio en el desarrollo de la especialización supone una gran oportunidad para incorporar estas competencias y conocimientos en el desarrollo de las funciones de los psicólogos educativos y mejorar la relevancia de sus aportaciones en la mejora de los procesos de enseñanza-aprendizaje, asesorando eficazmente tanto a profesores como a estudiantes y familias. Este es uno de los retos que a asumir urgentemente desde el ámbito académico y profesional.

\section{III- Conclusiones.}

\section{1.- Las alternativas: La mejora de los procesos de formación ligadas a las competencias}

La reflexión sobre las líneas de futuro de la psicología de la educación nos conduce inevitablemente a plantearnos la mejora de los procesos de formación de estos profesionales. Esta cuestión resulta imprescindible, dado que todo proceso de formación en una profesión redunda en la definición de perfiles de actuación y en la oferta de mejores servicios a la sociedad. Aunque hay que reconocer que las competencias individuales se pueden adquirir a través de diferentes experiencias formativas y profesionales, resulta necesario diseñar procesos de formación para el desempeño eficaz y de calidad de las competencias del psicólogo de la educación (Lionetti, Snyder, Christner \& McLaughlin, 2011). Y estamos convencidos de que este es el camino a recorrer en el momento actual si el objetivo es configurar la identidad de estos profesionales en el sistema educativo y asegurar sus aportaciones en la mejora de la calidad de la educación.

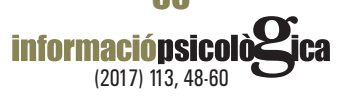


Desde el punto de vista académico, desde nuestra perspectiva, las sucesivas reformas de los Planes de Estudio del título de Psicología han mejorado notablemente la formación en aquellos dominios competenciales que son determinantes para ejercer la función de psicólogo educativo y que en el pasado resultaban claramente insuficientes (o inexistentes) para tal fin. En este sentido, en nuestro contexto más próximo de la Universidad de Valencia, basta con analizar y comparar el plan de estudios de la extinta licenciatura de Psicología con el actual título de grado para comprobar esta afirmación, dada la incorporación de materias obligatorias que abordan competencias y conocimientos previamente ausentes en los planes de estudio (p.e., trastornos del desarrollo) o que sólo se podían cursar de forma optativa (p.e., dificultades del aprendizaje), la ampliación temporal de materias relacionadas con psicología del desarrollo, o la generación de un itinerario formativo específico en este ámbito. Sin embargo, también resulta obvio que los planes de estudio actuales no permiten obtener una formación sólida de cara al ejercicio profesional como psicólogo de la educación (pese al carácter profesionalizante del grado), dado que no integra una formación suficientemente amplia y profunda para desarrollar las competencias implicadas en el ámbito profesional. En este punto conviene recordar que, a diferencia del caso español, la reforma de los planes de estudio de Psicología en los distintos países de Europa (p.e., Francia, Italia) contempló un modelo de grado de tres o cuatro años, no especializado, que no capacita para el ejercicio de la profesión y un postgrado con diversos másteres específicos (al menos en Psicología Educativa, Psicología Clínica y Psicología del Trabajo), que tienen como finalidad la adquisición de una formación avanzada, orientada a la especialización académica y/o profesional, o bien a promover la iniciación en tareas investigadoras.

Pero para que podamos alcanzar este estatus y reivindicar el reconocimiento de la especialización, tenemos que ponernos de acuerdo en cuáles serían las competencias - "unidades elementales de capacitación para el desempeño de un proceso de trabajo específico" (RD 797/1995)- de estos especialistas y que, a su vez, los diferenciarían de las otras especialidades reconocidas. Este proceso de delimitar competencias nos obligaría, en primer lugar, a delimitar los roles, funciones y tareas que deben realizar los orientadores y, en segundo lugar, a determinar las competencias requeridas y el alcance de las mismas, junto con los conocimientos, habilidades y actitudes correspondientes.

En el caso de los psicólogos de la educación, nos referimos a la capacidad de aplicar conocimientos, destrezas y actitudes al ejercicio de la psicología de la educación, incluyendo la capacidad de respuesta a problemas, la autonomía, la flexibilidad, la colaboración con los diferentes agentes y contextos de la intervención. Las competencias básicas de estos profesionales proporcionarían una descripción de los distintos roles a desempeñar por el psicólogo educativo relacionado con las principales áreas de intervención: dificultades del aprendizaje, atención a la diversidad, prevención, desarrollo, proceso de enseñanza aprendizaje y orientación y asesoramiento personal, académico y profesional. Y la determinación del perfil competencial de los orientadores que responda a las demandas actuales debería ir acompañado de un análisis de las implicaciones para su formación y acreditación profesional. 


\section{2.- ¿Y cuáles son las alternativas?}

Partimos de la consideración de que la complejidad de las tareas de orientación nos lleva a valorar la necesidad de que existan servicios de orientación formados por equipos multidisciplinares donde los psicólogos sean integrantes esenciales de los mismos. Para ello, su formación debe ser técnica, específica y prolongada, lo que nos conduce a valorar la conveniencia de diseñar una propuesta formativa de postgrado pensada para el psicólogo educativo con una estructura que, sería deseable, compartieran todas las universidades. Para abordar esta cuestión relevante consideramos que la mejor alternativa, tal y como hemos apuntado con anterioridad, debería consistir en abordar el análisis de las competencias del psicólogo de la educación y llegar a un consenso entre todos los implicados -Administración Educativa, Universidad, Asociaciones Profesionales-. El acuerdo sobre las competencias sería un primer paso que nos permitiría superar algunas de las cuestiones polémicas y controvertidas que hemos recogido en estas páginas. Es decir, contestar a las cuestiones relativas a ¿qué hace un psicólogo de la educación?, ¿cómo interviene?, ¿cuáles son sus prioridades de actuación? Partir de este acuerdo favorecería, no tenemos duda, el consenso sobre la formación de estos psicólogos y el acuerdo sobre un máster específico que garantizara la adquisición de las competencias para el desempeño de las tareas que le resultarían propias.

Dicho de otro modo, se trataría de "especializar" la orientación educativa. Un master en Psicología de la Educación exclusivo para graduados/licenciados en psicología que ampliara el foco, desarrollando competencias para un amplio volumen de tareas profesionales educativas que ya vienen desempeñando los psicólogos (por supuesto, todas las instruccionales y relacionadas con la evaluación e intervención en dificultades del aprendizaje y trastornos del desarrollo) a las que habría que incorporar otras que resultan necesarias en el desempeño de este puesto de trabajo y que no se abordan en la formación del grado en Psicología (ni en el actual master de Formación de Profesorado de Secundaria), permitiendo también el acceso a servicios especializados relacionados con el diagnóstico e intervención psicoeducativa en contextos escolares.

Este acuerdo implicaría, por sí solo, algunos cambios necesarios en el panorama actual. A modo de ejemplo, las Administraciones Educativas deberían introducir cambios en la legislación para actualizar las funciones y competencias de los orientadores a partir de los acuerdos adoptados. A partir de aquí, establecer definir y consensuar el proceso formativo -máster- que habilitaría para la adquisición del marco de competencias del orientador.

\section{3.- ¿Una o varias psicologías? La problemática derivada de la regulación parcial de la profesión}

Pero la perspectiva y apuesta destacada en los párrafos precedentes no aborda el segundo gran foco de problemáticas ligadas a la propuesta formativa de base y al ejercicio profesional de la psicología de la educación: la enorme asimetría actual en el campo de la psicología provocada por la regulación de la figura del Psicólogo General Sanitario, dado que la perspectiva previamente destacada aboga por el reconocimiento de las competencias de los Psicólogos de la Educación para evaluar e intervenir en el campo de las dificultades del aprendizaje y de los trastornos del desarrollo, dentro y fuera del sistema escolar.

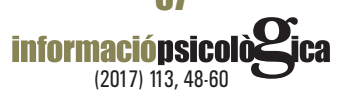


En el marco organizativo actual de las enseñanzas universitarias, pensamos que la solución a esta problemática pasaría, entre otras consideraciones, por plantearse la reorganización de la formación de postgrado en el ámbito de la psicología, alejándonos de la perspectiva actual que prima la asimetría, pero también distanciándose de un afán regulatorio desaforado que abogara por establecer múltiples itinerarios formativos cuasi-paralelos entre los distintos ámbitos profesionales de la psicología. De otro modo, las preguntas pueden ser ¿queremos mantener unos masters especializados que mantengan una cierta convergencia ente sí y que faciliten la actualización y reorientación de las carreras profesionales de nuestros egresados o buscamos caminos totalmente diferenciados tras el grado de psicología (o incluso dentro del mismo grado) que requieran ingentes costes de tiempos y esfuerzo para poder reorientar la vida profesional? De otro modo más directo, y centrándonos en el momento actual ¿cuál es el calificativo al que queremos dar mayor peso en el MPGS, a general o a sanitario?

Como se indica en el artículo introductorio, nos guste o no, la normativa de rango superior nos impone una doble exigencia, para ser orientador es necesario cursar el master de formación del profesorado (a todas luces insatisfactorio en su versión actual tal como se ha hecho referencia previamente), pero si "las evaluaciones e intervenciones psicológicas (...) que influyen en la promoción y mejora del estado general de la salud" quedan reservadas para quienes cursan el MPGS, ¿esto no puede llevarnos a unas luchas intestinas dentro del propio campo de la psicología y, en un futuro no muy lejano, sobre a quién corresponden las competencias profesionales relacionadas con la evaluación e intervención en el ámbito de los trastornos del desarrollo y de las dificultades del aprendizaje?, ¿esto no podría justificar que muchos de los problemas de diagnóstico e intervención psicoeducativa requieran la remisión a servicios externos de salud mental y servicios sociales que efectúen valoraciones descontextualizadas, sin compartir significados ni propuestas de actuación con los profesionales educativos?, ¿no estaremos así apoyando que las competencias específicas de los psicólogos educativos sean cada vez más ajenas a los servicios de orientación y frustremos cada vez más las expectativas de familias y profesores que depositan su confianza en los mismos? Y, por último, ¿no supondrá una forma indirecta de derivar problemáticas educativas no sólo a otras administraciones públicas sino también hacia servicios privados a los que deban dirigirse las familias?

Centrándonos estrictamente en la definición de itinerarios académicos, en la próxima regulación de los créditos sanitarios que debe incorporar el grado de psicología, siempre que persista la actual estructura y organización de los títulos de grado ¿resultaría razonable integrar en la misma las competencias propias de Psicología del Desarrollo y de la propia Psicología de la Educación? Y, por otro lado, ¿podría replantearse una profunda reorganización del MPGS que se centrara más en el término psicólogo general que en el de psicólogo sanitario y que permitiera su compatibilización con una especialización real en Psicología de la Educación (o en otros ámbitos de la psicología) plausible en términos de tiempos y de esfuerzos -personal y económico? Pensamos que esta cuestión evitaría gran parte de las tensiones actuales (ya conflictos declarados en algunos casos) entre las diferentes especializaciones profesionales de la psicología, siempre que se facilitara a nuestros estudiantes y egresados poder reconducir sus campos de actuación en función de los avatares que acon-

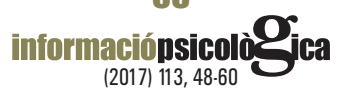




\section{dossier}

tezcan en sus vidas profesionales, y facilitaría la cohesión (en parte importante ya perdida en estos últimos años) entre los ámbitos disciplinares y profesionales de la psicología. No es tarea sencilla pero, mientras persista la situación actual en la estructura y organización de los estudios universitarios, otras opciones suponen para nuestros graduados toma de decisiones con difícil retorno, problemas importantes para reconducir sus planes de carrera, e importantes conflictos de intereses entre áreas de conocimiento y campos profesionales de la psicología de los que nadie puede anticipar hacia donde se dirigirán y, lo más importante, dónde pueden acabar. El camino, al menos en gran parte, está en nuestras manos.

\section{Referencias}

Acuerdo sobre la Inserción del Psicólogo Educativo en el Sistema de Educación Español no Universitario. Infocop, 2010.

Coll, C. (1988). Conocimiento Psicológico y práctica educativa. Barcelona: Barcanova.

Coll, C. (1989). Psicología Académica y Psicología Profesional en el Campo de la Educación. Anuario de Psicología, 41, 49-73.

Coll, C. (1991). Más allá de las etiquetas: Convergencia disciplinar, solapamiento profesional y necesidades de formación en la intervención psicopedagógica. Papeles del Psicólogo, 51, 63-76.

Coll, C. (1996). Psicopedagogía: Confluencia Disciplinar y Espacio Profesional. En C. Monereo e I. Solé (Eds), El Asesoramiento Psicopedagógico: una Perspectiva Profesional y Constructivista, pp. 33-52. Madrid: Alianza.

Ervin, R.A., Peacock, G., \& Merrel, K.W. (2010). The School Psychologist as a Problem Solver in the 21 st. Century. In G. Gimpel, R. A. Ervin, E.J. Daly, \& K.W. Merrel (Eds), Practical Handbook of School Psychology: Effective Practices for the 21 st. Century, pp. 3-12. New York: The Guilford Press.

Fagan, T.K. y Wise, P.S. (1994): School Psychology: Past, Present, and Future. New York: Longman.

Fagan, Th.K. \& Wise, P.S. (2000): School Psychology: Past, Present and Future. Bethesda: Kluwer AcademicNational Association of School Psychologists.

Fernández, J. (2011). La especificidad del Psicólogo Educativo. Papeles del Psicólogo, 32, 247-253.

García-Ros, R. y Pérez-González, F. (1996). Delimitación de prioridades en las áreas de actuación del psicólogo escolar: Un análisis de las intervenciones con profesores, estudiantes, padres y coordinación de los equipos multiprofesionales. En Psicología del Desarrollo y de la Educación. Intervención psicoeducativa, pp. 293-300. Sevilla: Eudema.

Gilman, R. \& Gabriel, S. (2004). Perceptions of School Psychological Services for Educational Professionals: Results from a Multi-State Survey Pilot Study. School Psychology Review, 33(2), 271-286.

Gilman, R., Misall, K., and Macks, R. (2011). Emerging Trends in the Preparation of School Psychologists for Practice. In M. A. Bray \& T.J. Kehle, (Eds), The Oxford Handbook of School Psychology, pp. 774-785. New York: Oxford University Press.

Gutkin, T.B. \& Conoley, J.C. (1990). Reconceptualizing school Psychology from a service delivery perspective: Implications for practice, training, and research. Journal of School Psychology, 28, 203-224.

I Encuentro Nacional de Profesionales de la Psicología de la Educación (2009). Barcelona.

I.S.P.A. (International School Psychology Assocciation). http://www.ispaweb.org/en/index.html (Fecha de consulta: 9/05/16).

Lionetti, T.M., Snyder, E.P., Christner, R.W., \& McLaughlin, C.L. (2011). Developing and Enhancing Competencies in the Practice of School Psychology. In M. Bray, and T.J. Kehle (Eds), A Practical Guide to Building Professional Competencies in School Psychology, pp. 1-12. New York: Springer.

Mägi, K. \& Kikas, E. (2009). School Psychologists' Role in School. School Psychology International 30, 331-346. 


\section{dossier}

Manifiesto en defensa de la profesionalidad de la Orientación Educativa (2011)

Peretó, M. y de Vicente, A. (2014). Mapa profesional de la Psicología en los servicios de orientación educativa por Comunidades Autónomas - resultados preliminares. Infocop 18-06-2014.

Pérez-González, F. y García-Ros, R. (2011). La prestación de servicios psicológicos en los contextos escolares: Un análisis de las funciones más demandadas y la implicación de los profesionales en su desarrollo. VI Congreso Internacional de Psicología y Educación. Valladolid: Actas del Congreso.

Pérez-Solís, M. (2011). ¿Por qué un máster en Psicología Educativa? Psicología Educativa, 17, 101-114.

Pérez-Solís, M. (2011). La paradoja del profesor orientador. Papeles del Psicólogo, 32, 232-241.

Skalski, A. K.; Minke, K.; Rossen, E.; Cowan, K. C.; Kelly, J.; Armistead, R. \& Smith, A. (2015). NASP Practice Model Implementation Guide. Bethesda: National Association of School Psychologists.

Solé, I. y Colomina, R. (1999). Intervención psicopedagógica: una -¿o más de una?- realidad compleja. Infancia y Aprendizaje 87, 9-26. 